\title{
Specific Structural Analysis of Solanesol and Related Compounds by Fast Atom Bombardment in Combination with Tandem Mass Spectrometry*
}

by

Nancy J. Jensen and Terry Sumpter

Research Center, Philip Morris,

Richmond, VA, USA .

\section{SUMMARY}

Rapid identification of solanesol even in the presence of a complex matrix can be accomplished using fast atom bombardment (FAB) mass spectrometry in combination with tandem mass spectrometry methods. FAB MS analysis using a nitrobenzyl alcohol matrix doped with lithium yields an abundant $(M+L i)^{+}$ion of $m / z$ of 637 which is a very characteristic marker for the presence of solanesol and is quite sufficient for screening for the presence of solanesol in many cases. MS/MS analysis of this ion produces a spectrum which yields specific structural information regarding each isoprene unit of the entire structure. Furthermore, these methods are very useful for characterizing unknowns as demonstrated by the characterization of diol, triol and ester impurities in commercially available solanesol. Application of the MS/MS/MS analysis provides insight into the frag. mentation mechanism and can be a useful alternative to conventional labeling methods for confirming spectral interpretations.

\footnotetext{
* Received: 12 January 1994 - accepted: 7. December 1994
}

\section{ZUSAMMENFASSUNG}

Solanesol kann auch in Gegenwart einer komplexen Matrix mit 'Fast Atom Bombardment' (FAB)Massenspektrometrie, kombiniert mit Tandemmassenspektrometischen Methoden schnell identifiziert werden. Mit FAB-Massenspektrometrie unter Verwendung einer Nitrobenzyl-Alkohol-Matrix, die mit Lithium versetzt ist, erhält man ein $(M+L i)^{+}$-Ion mit einem Masse/Ladungsverhältnis $(\mathrm{m} / \mathrm{z})$ von 637 . Dies ist ein charakteristischer Marker für die Anwesenheit von Solanesol und ist für ein Screening in vielen Fällen ausreichend. Die MS/MS-Analyse dieses Ions ergibt ein Spektrum, das beziiglich jeder Isopreneinheit spezifische Informationen über die gesamte Molekuullstruktur liefert. Darüber hinaus sind diese Methoden sehr nützlich bei der Charakterisierung unbekannter Substanzen, wie es bei Diol-, Triol- und Esterverunreinigungen im handelsüblichen Solanesol gezeigt wurde. Die MS/MS/MS-Analyse gibt Aufschluß über die Fragmentierungsmechanismen und kann als nützliche Alternative zu konventionellen Markienungsmethoden zur Bestätigung spektrometrischer Interpretationen dienen. 


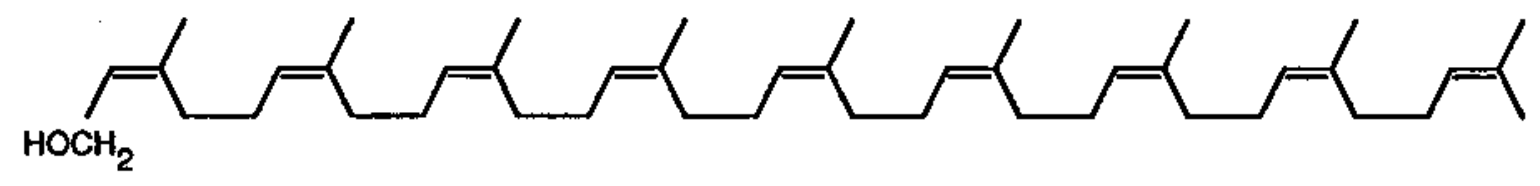

\section{RESUME}

Le solanesol peut rapidement être identifié mêtme en présence d'une matrice complexe au moyen de la spectrométrie de masse 'fast atom bombardment' (FAB), combinée de méthodes de spectrométrie de masse tandem. Au moyen de la spectrométrie de masse $\mathrm{FAB}$ en présence d'une matrice en alcool nitrobenzylique additionnée de lithium on obtient un ion $(\mathrm{M}+\mathrm{Li})^{+}$avec un rapport masse/charge de 637 , ce qui est un indiquateur très caractéristique de solanesol et est souvent suffisant pour étudier la présence du solanesol. L'analyse MS/MS de cet ion fournit un spectre qui donne des informations spécifiques de chaque unité d'isoprènes composant la structure d'ensemble. En outre, ces méthodes sont très utile pour caractériser les substances inconnues, ce qui a été montré à propos d'impuretés de dioles, trioles et d'esters dans le solanesol commercial. L'analyse MS/MS/MS donne des renseignements sur les méchanismes de fragmentation et peut servir d'analyse utile employée aux lieux de méthodes de marquages conventionnelles pour confirmer les interprétations spectrométriques.

\section{INTRODUCTION}

The analysis of solanesol has been a challenging problem for tobacco chemists for many years for two important reasons. Firstly, solanesol (see structure 1) is considered to be a compound characteristic of tobacco and related species and is not widely distributed in nature; hence, facile analyses would be very useful in a number of applications in tobacco related investigations. Secondly, solanesol is difficult to analyze by conventional GC (gas chromatography) or GC-MS (gas chromatography - mass spectrometry methods). The size of the molecule presents challenges for GC analyses and the isoprene chain fragments extensively in conventional electron impact (EI) mass spectrometry to yield a very low abundance molecular ion at best and many low mass fragments which reveal little information regarding specific structural features of the alkyl chain.

In recent years fast atom bombardment (FAB) ionization in combination with tandem mass spectrometry has become an established method for specific structural characterization of large polar labile molecules both as pure substances and as components of complex mixtures
$(1,2)$. This method is especially useful for many kinds of molecules which are not readily amenable to conventional EI analysis and hence would be expected to be applicable to the analysis of solanesol. Typically fast atom bombardment produces a molecular ion species, usually $(\mathrm{M}+\mathrm{H})^{+}$ions in the positive ion mode or $(\mathrm{M}$ $\mathrm{H})^{-}$ions in the negative ion mode for very polar species. For less polar species such as alcohols the addition of lithium ions as a dopant to the sample facilitates the formation of $(\mathrm{M}+\mathrm{Li})^{+}$ions which are readily desorbed in the $\mathrm{FAB}$ process and are species that are characteristic of the molecular ion.

Since fragmentation is often quite limited in the FAB analysis, it is frequently combined with the tandem (MS/MS) experiment to provide detailed structural information. Fragmentation in the MS/MS analysis, which results from collision of a closed shell ion such as $(M+$ $\mathrm{H})^{+},(\mathrm{M}-\mathrm{H})^{-}$or $(\mathrm{M}+\mathrm{Li})^{+}$with a noble gas, is often quite different than that observed when an open shelled ion $\left(\mathrm{M}^{+}\right)$decomposes in conventional electron impact mass spectrometry. Charge-remote fragmentation is one example of a fragmentation that is not observed in electron impact mass spectrometry but is a characteristic fragmentation of closed-shelled long-chained aliphatic compounds subjected to collisional activation (3).

Charge-remote fragmentations are so named because the gas-phase decompositions appear to occur at sites that are physically removed from the presumed location of the charge. This fragmentation most likely involves a series of parallel reactions with highly specific 1,4-elimination of $\mathrm{H}_{2}$ and neutral alkene loss (3). This process generates a series of fragments which begin at the alkyl terminus and proceed along the entire chain thus providing a very specific structural fingerprint for the entire chain. If a modification such as a branch or a double bond occurs, the fragmentation is interrupted in a characteristic manner and the resulting fragmentation pattern can be interpreted to identify both the type and location of the structural modification.

Because solanesol is very similar to the types of molecules which are known to be amenable to $\mathrm{FAB}$ analysis and MS/MS analysis provides the possibility of obtaining detailed specific structural information for the entire alkyl chain, this investigation was undertaken to determine if FAB in combination with tandem mass spectrometry could be used as a substantially improved method for rapid detection and specific structural characteri- 
zation of solanesol. Furthermore since the ion of interest in the MS/MS analysis is selected and separated from the other source generated ions in the first stage of the experiment, the method is potentially applicable for obtaining this precise and detailed information from complex mixtures without prior purification steps.

\section{EXPERIMENTAL METHOD}

Solanesol samples were obtained from both Sigma Chemical Company (St. Louis MO) and in house extraction of tobacco for use in this investigation of enhanced methods for structural characterization of solanesol and related compounds.

Mass spectral analyses were performed using a JEOL SX 102/ SX 102 tandem mass spectrometer system. This is a four sector instrument in which both MS I and MS II are double focusing reverse geometry instruments. The instrument is based on ion optics developed by Matsuda and is designed with quadrupole lensing for enhanced transmission and sensitivity (4). The instrument is equipped with two collision cells; one in the first field free region and the second at the interface between MS I and MS II.

For all experiments positive ion fast atom bombardment (FAB) ionization with xenon at $6 \mathrm{KeV}$ and $10 \mathrm{~mA}$ emission was used as the ionization mode. Both liquid and solid samples were treated with a nitrobenzyl alcohol (NBA) matrix doped with lithium and deposited on the $F A B$ probe tip for direct probe $F A B$ analysis. The full accelerating voltage of $10 \mathrm{KeV}$ was used except as noted below. All data were collected using the DA 6000 data system.

Four types of mass spectral experiments were performed with the FAB generated ions. They are as follows:

$F A B$ experiment. Full scan $\mathrm{FAB}$ mass spectra were obtained by scanning the magnet of MS I over the mass range of 1 to $1000 \mathrm{u}$ at a scan slope of $0-2300 \mathrm{u} / 60 \mathrm{sec}$. A resolving power of ca. 1000 was used and the FAB spectra were recorded by the detector of MS I in a scan acquisition mode.

High resolution $F A B$ experiment. High resolution FAB mass spectra were obtained by an EF (voltage) scan of MS I over a narrow mass range. A resolving power of 10,000 was used and the spectra were recorded by the detector of MS I in an accumulation acquisition mode. For accurate mass assignment the sample was placed on one side of a rotatable double-sided probe tip and cesium iodide calibrant was placed on the other side of the probe tip. During the course of the experiment the tip was rotated on alternate scans to permit nearly simultaneous acquisition of sample and calibrant spectra.
$M S / M S$ experiment. For the tandem (MS/MS) experiment, the $(\mathrm{M}+\mathrm{Li})^{+}$parent ion was selected by fixing the magnet and ESA of MS I to pass only the desired ion, collisionally activating the ion with helium at ca. 75\% beam reduction in the collision cell between MS I and MS II, and then scanning MS II in a B/E scan to obtain the first generation product ion spectrum. The collision cell was floated at $7 \mathrm{KeV}$ to minimize discrimination against low mass ions. The MS/MS spectra were recorded by the detector of MS II in an accumulation mode and are typically an average of 3-5 scans.

$M S / M S / M S$ Experiment. For the MS/MS/MS experiment, $(\mathrm{M}+\mathrm{Li})^{+}$ions generated in the ion source were collisionally activated with helium at ca. $75 \%$ beam reduction in the first field free region (immediately after the ion source) and the first generation product ion of interest was selected using MS I. The magnet and the ESA of MS I were independently set to fixed positions to pass an ion of only the mass and energy of the first gener. ation product ion of interest. Although source generated ions of the same mass as the desired product are formed, the energy imparted to a fragment ion formed by collision is proportional to the ratio of the mass of the fragment to the parent ion. The selected first generation product ion was passed from MS I to the interface between MS I and MS II, collisionally activated with helium at 60-70\% beam reduction and second generation product ions were analyzed with a $B / E$ linked scan of MS II. The MS/MS/MS spectra were recorded by the detector of MS II in an accumulation mode and are typically an average of 10-15 scans. Two instrumental modifications were required to accomplish this. The first was direct monitoring of the ESA voltage of MS I to three decimal places. The second was the addition of a switch to facilitate decoupling of the ESA and magnet of MS I.

\section{RESULTS}

The initial motivation for this work was to develop a facile method for specific characterization of solanesol which would be suitable for rapid identification of solanesol in the presence of complex mixtures. As Figure 1a shows analysis of solanesol by FAB in a matrix of NBA doped with $\mathrm{Li}^{+}$yields an abundant molecular ion species $(\mathrm{M}+\mathrm{Li})^{+}$of $\mathrm{m} / \mathrm{z}$ of 637 (nominal mass). No appreciably abundant ion characteristic of the intact solanesol molecule is observed unless the lithium dopant is added. This is quite fortuitous as the presence of solanesol in a complex mixture is readily indicated if the mixture is first analyzed by FAB in an NBA matrix, then treated with $\mathrm{Li}^{+}$and examined again. The appearance of the ion of $\mathrm{m} / \mathrm{z}$ of 637 in the spectrum obtained from the second part of the analysis indicates the presence of sola- 


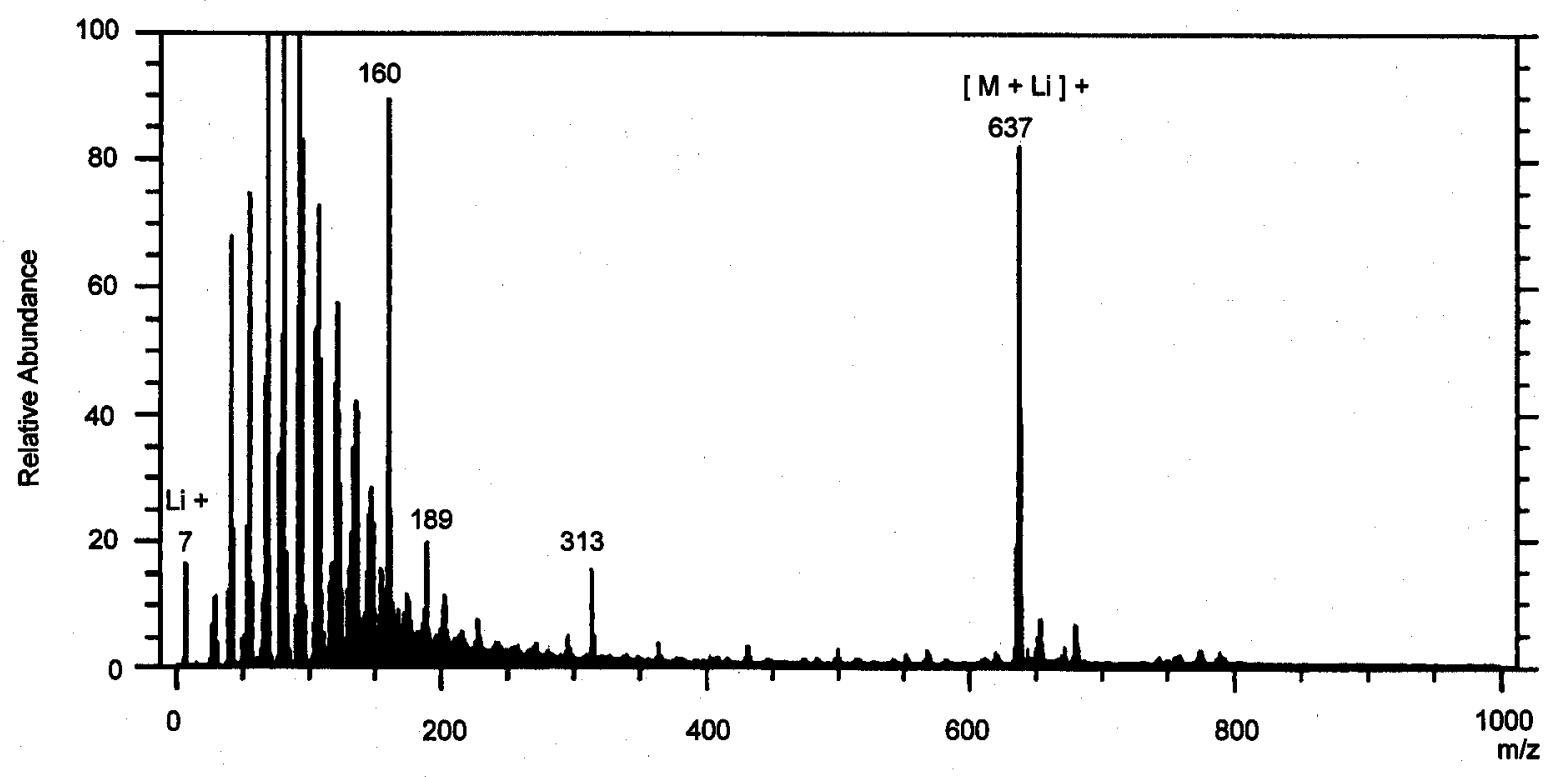

Figure 1a.

FAB mass spectrum of solanesol in an NBA matrix doped with $\mathrm{Li}^{+}$.

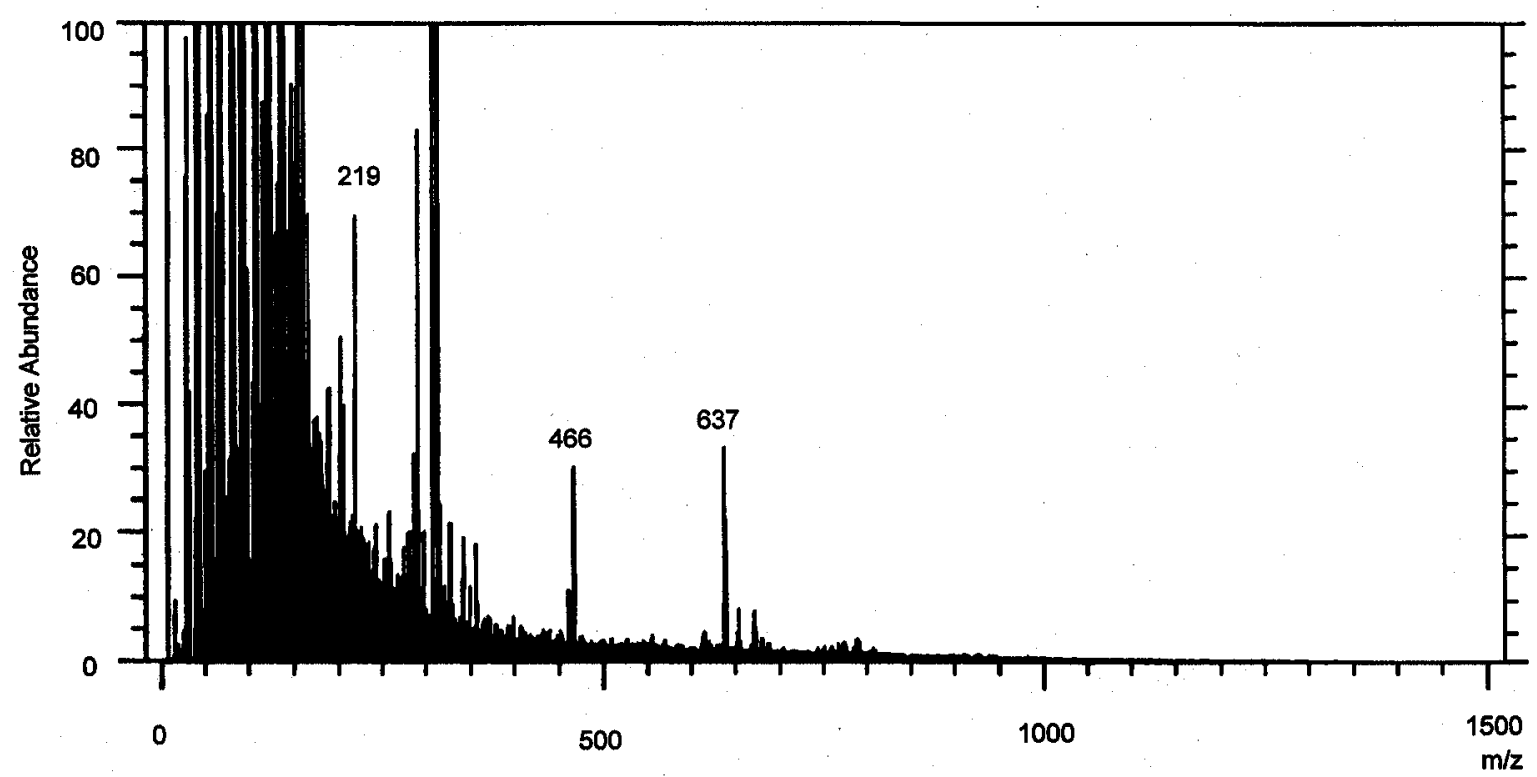

Figure 1b.

FAB mass spectrum obtained from direct analysis of solanesol spot on paper treated with an NBA matrix doped with $\mathrm{LI}^{+}$. 
nesol.

The applicability of the analysis to a complex system is readily demonstrated by deliberately spotting cigarette paper with a methylene chloride solution of solanesol, excising the spotted area and directly analyzing the spotted piece of paper by FAB using the NBA matrix with lithium dopant. Although a number of abundant low mass ions are observed from the paper matrix, the $(\mathrm{M}+\mathrm{Li})^{+}$ion of $\mathrm{m} / \mathrm{z}$ of 637 of solanesol is readily distinguished (see Figure 1b). This method is quite suitable for a rapid qualitative survey for solanesol; however the ultimate sensitivity of the process is dependent on the complexity and nature of sample.

Selection of the molecular ion species $(\mathrm{M}+\mathrm{Li})^{+}$with $\mathrm{MS}$ $\mathrm{I}$, collisional activation, and subsequent analysis of the product ions with MS $\amalg$ yielded a product ion spectrum rich in structural information for the, solanesol ion (Figure 2). The principal ions of the spectrum are an ion formed as the result of the loss of water and a series of product ions which result from charge-remote fragmentation. This type of fragmentation is well documented elsewhere (see references 3 and 5 for reviews of the topic). In this type of fragmentation product ions are formed as the result of a series of specific cleavages with $\mathrm{H}_{2}$ elimination along the alkyl chain. Fragment $A$ in Figure 2 is formed as a result of the loss of a terminal $\mathrm{CH}_{4}$, fragment $B$ results from the loss of the terminal $\mathrm{C}_{5} \mathrm{H}_{10}$, fragment $\mathrm{C}$ results from the loss of the terminal $\mathrm{C}_{10} \mathrm{H}_{18}$... etc. Thus, the fragmentation can be correlated with specific cleavages of the hydrocarbon chain as shown in Figure 2 and in effect provides a "fingerprint" spectrum for the entire solanesol terpene chain.
The sample which yielded the spectrum shown in Figure 1 is "pure" solanesol obtained from the commercial supplier. The ion of $\mathrm{m} / \mathrm{z}$ of $637(\mathrm{M}+\mathrm{Li})^{+}$is indeed the most prominent high mass ion. However, if one examines the spectrum carefully, one notes the presence of other ions of $\mathrm{m} / \mathrm{z}$ of 679,671 and 653 . The presence of these ions higher in mass than the molecular ion suggests that impurities are present in the sample. A sample of solanesol prepared and purified by conventional extraction procedures in house yielded a similar spectrum. These ions are quite interesting as they differ from solanesol by masses of 16,34 , and 42 and thus have the potential of close relationship to solanesol. Conventional separation procedures may not separate very closely related species. It should be noted that although matrix artifacts are sometimes observed in FAB analyses, these masses are not consistent with such species.

Since the FAB MS/MS analysis yields abundant specific information for solanesol, we explored FAB MS/MS analysis of the impurities in "purified" solanesol. Collisional activation of the ion of $\mathrm{m} / \mathrm{z}$ of 679 yields the principal fragmentation of the loss of $\mathbf{4 2}$ to form the ion of $\mathrm{m} / \mathrm{z}$ of 637 (Figure 3a). Although much less abundant than the ion of $\mathrm{m} / \mathrm{z}$ of 637 , a series of ions exactly like that found in the MS/MS spectrum of solanesol is also observed if the region of the spectrum from $\mathrm{m} / \mathrm{z} 90$ to $\mathrm{m} / \mathrm{z} 430$ is magnified (see inset in Figure 3a). These data indicate that the compound which forms the ion of $\mathrm{m} / \mathrm{z}$ of 679 is an acetate ester of solanesol.

The MS/MS spectrum of the ion of $\mathrm{m} / \mathrm{z}$ of 653 is characterized by a series of doublets differing in mass by $16 \mathrm{u}$ (Figure 3b). The lower mass series of ions of this set

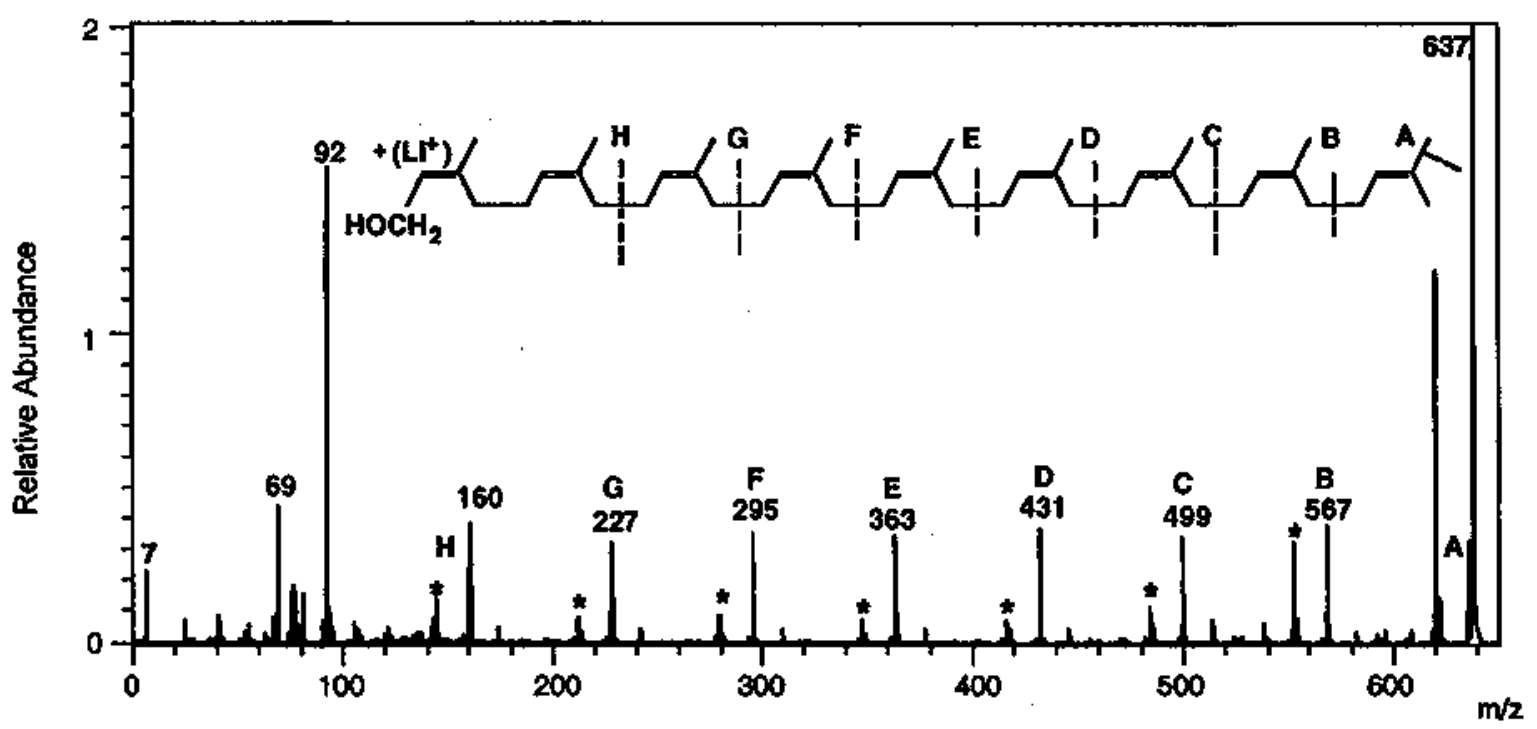

Figure 2.

FAB MS/MS spectrum of the $(M+L)^{+}$lon of $\mathrm{m} / \mathrm{z}$ of 637 of solanesol. 

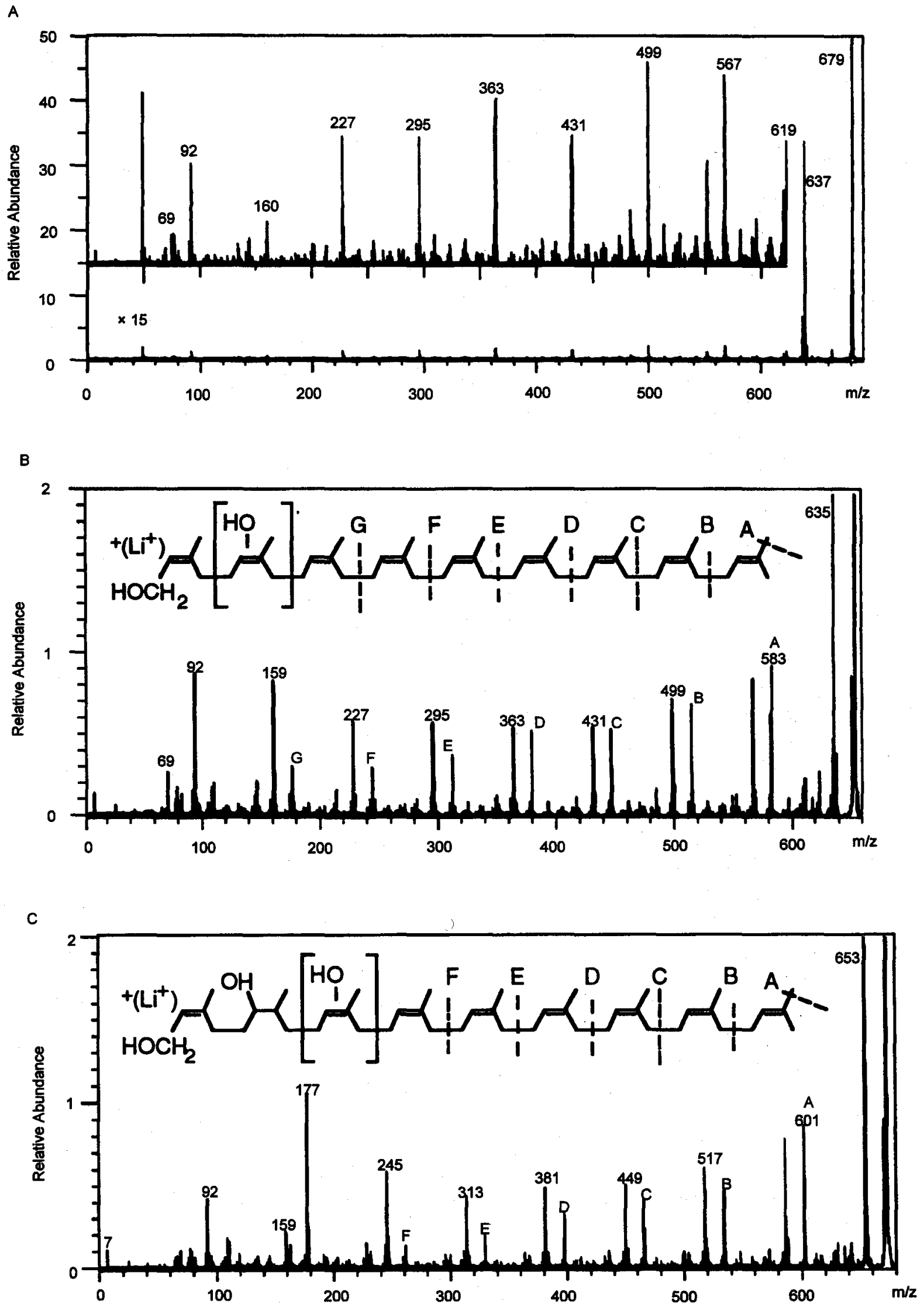

Figure 3.

FAB MS/MS spectra of the impurity lons of (a) $\mathrm{m} / 2679$, (b) $\mathrm{m} / \mathrm{z} 653$, and (c) $\mathrm{m} / \mathrm{z}$ of 671 . 
Table 1.

Solanesol M/Z 637 $(\mathbf{M}+\mathbf{L i})^{+}$
Diol $M / Z 653$

$\left(\mathrm{M}+\mathrm{Li}-\mathrm{H}_{2} \mathrm{O}\right)^{+}(\mathrm{M}+\mathrm{Li})^{+}$
Triol $M / Z 671$

$\left(M+\mathrm{LI}-\mathrm{H}_{2} \mathrm{O}\right)^{*}(\mathrm{M}+\mathrm{LI})^{+}$

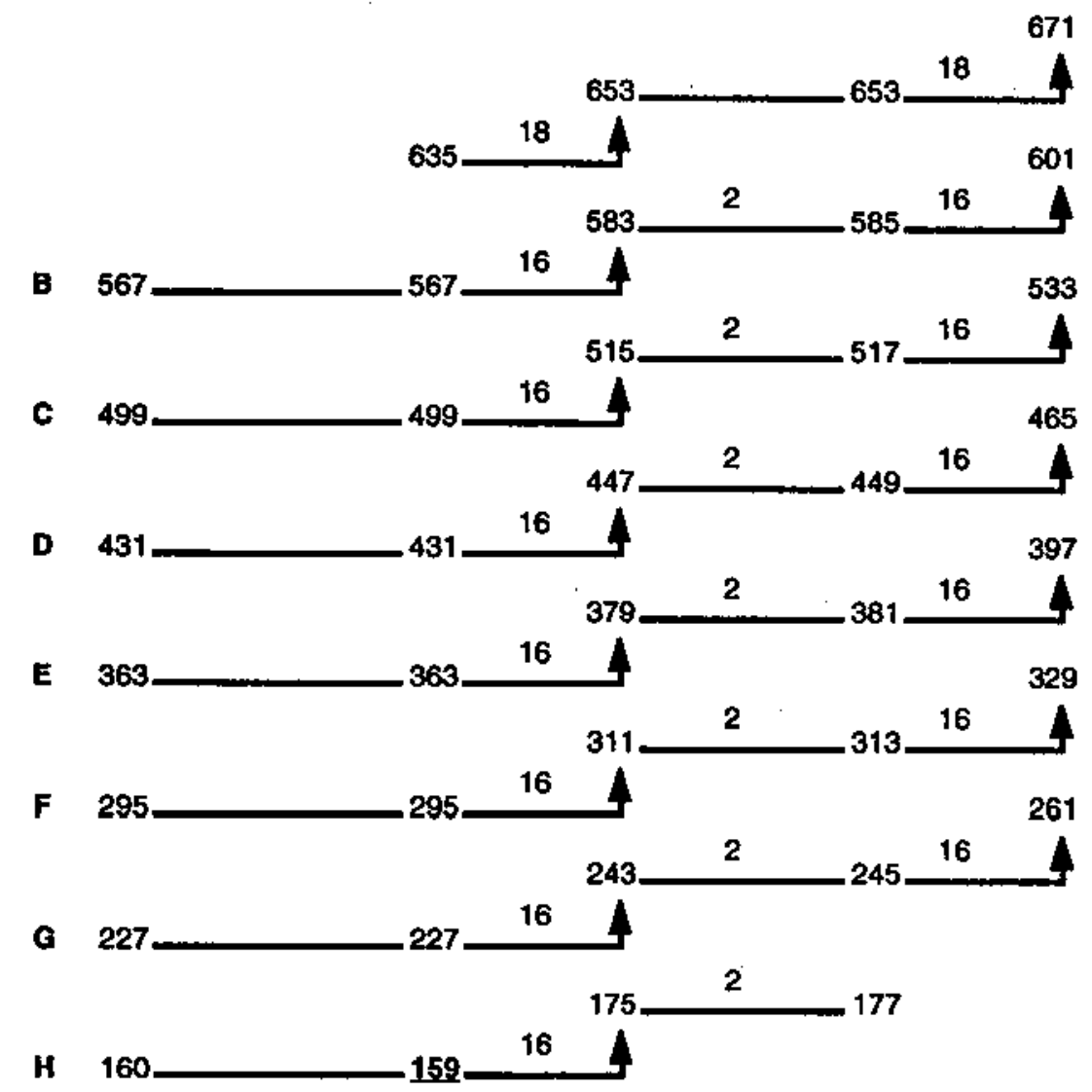

of doublets (ion of $\mathrm{m} / \mathrm{z}$ of $635,567,499,431,363,295$, 227 and 159) is like that of solanesol except that the last ion of the series is the ion of $\mathrm{m} / \mathrm{z}$ of 159 instead of 160 as it is for solanesol. The fact that the higher mass mass series of ions in the doublets differs by $16 \mathrm{u}$ suggests that the compound is a diol. High resolution mass spectrometry confirmed the presence of an additional oxygen as the exact mass was determined to be 653.5859 for this ion which corresponds to a formula of $\mathrm{C}_{45} \mathrm{H}_{74} \mathrm{O}_{2} \mathrm{Li}$. The higher mass series of ions and the key ion of $\mathrm{m} / \mathrm{z}$ of 159 suggest that a second hydroxy group is present in the second isoprene unit (see structure Figure 3b). The exact position of the $\mathrm{OH}$ in the second isoprene unit cannot be assigned but the mass of the ion of $\mathrm{m} / \mathrm{z}$ of 175 does indicate that it is not an $\mathrm{H}_{2} \mathrm{O}$ addition to the double bond. The lower mass series of ions in the series of doublets in the MS/MS spectrum of $\mathrm{m} / \mathrm{z}$ of 653 may be accounted for by loss of water with subsequent shifting of the double bonds followed by charge-remote fragmentation of the resulting ion. Again, the ion of $\mathrm{m} / \mathrm{z}$ of 159 is a key ion. For solanesol or the acetate of solanesol in which the isoprene chain is not modified, the corresponding ion is the ion of $\mathrm{m} / \mathrm{z}$ of 160 .

High resolution mass spectral analysis of the ion of $\mathrm{m} / \mathrm{z}$ of 671 revealed an exact mass of 671.5882 which corresponds to a formula of $\mathrm{C}_{45} \mathrm{H}_{76} \mathrm{O}_{3} \mathrm{Li}$. The MS/MS spectrum of this ion reveals that it is most likely a triol (see Figure 3c). Again, as for the diol, a series of doublets is observed with the doublet ions separated by $16 \mathrm{u}$. The lower mass series of ions (ions of $\mathrm{m} / \mathrm{z}$ of $585,517,449$, $381,313,245$ and 177) parallels the higher mass series of ions of the diol offset by $2 u$. The general similarity of this spectrum to that for the ion of $\mathrm{m} / \mathrm{z}$ of 653 and the exact mass data which show three oxygens suggests that the compound is most likely a triol. The substantial differences between this spectrum and the spectrum of the ion of $\mathrm{m} / \mathrm{z}$ of 653 in the range of $\mathrm{m} / \mathrm{z}$ of 100 to 250 indicate that the second and third isoprene units bear the additional oxygens. The offset of two mass units and the ion of $\mathrm{m} / \mathrm{z}$ of 177 suggests that the $\mathrm{OH}$ of the second 
isoprene unit is an $\mathrm{H}_{2} \mathrm{O}$ addition at the double bond. Again, the precise location of the oxygen in the second and third isoprene units can not be assigned and, as explained above, the set of doublets can be accounted for by charge-remote fragmentation of the entire ion and elimination of $\mathrm{H}_{2} \mathrm{O}$ followed by charge-remote fragmentation. The relationship of the fragmentation patterns for the $(M+L i)^{+}$ion of solanesol and the ions of $m / z$ of 653 and 671 is summarized in Table 1.

The $\mathrm{MS}^{3}$ spectrum of a product ion from the $(\mathrm{M}+\mathrm{Li})^{+}$ ion of lithiated solanesol yields considerable mechanistic insight. The principal ions of the MS/MS spectrum of solanesol are quite interpretable as resulting from loss of water and charge-remote fragmentation of the alkyl chain (Figure 2).

Traditional exploration of this interpretation and the explanation of the origins of the other lesser abundance fragment ions would require preparation of labeled materials; however, MS/MS/MS analysis of first generation (MS/MS) product ions is a more direct and rapid alternative. The ion of $\mathrm{m} / \mathrm{z}$ of 619 in the MS/MS spectrum which is formed by the loss of water from solanesol is of suitable abundance for such analysis. Previous work would suggest that the abundance of this ion is substantially less than $0.5 \%$ of the parent ion beam (6). The second generation product ion (MS/MS/MS) spectrum does indeed have a series of charge remote fragmentation ions differing by the loss of water from the principal series of ions in the solanesol $\left(\mathrm{M}+\mathrm{Li}^{+}\right)$spectrum (Figure 4).

These data indicate that although the principal site attachment of lithium may be at the alcohol functionality per se, the formation of a lithium adduct in a complex with the double bond adjacent to the alcohol is also likely. Low abundance ions may be associated with lithium adducts with double bonds at other positions in chain. The fact that alteration in amount of lithium dopant can alter the respective relative abundances of ions observed in the MS/MS/MS spectrum of this ion further support for the concept of multiple sites of lithium attachment.

These data also confirms the origin of the lesser abundant series of ions in the MS/MS spectrum of solanesol (designated with * in Figure 2) as arising from charge remote fragmentation of the dehydrated solanesol. By virtue of the manner in which the MS/MS/MS experiment is performed, all ions observed in the MS/MS/MS spectrum must be formed from an ion of $\mathrm{m} / \mathrm{z}$ of 619 which was formed from the decomposition of an ion of $\mathrm{m} / \mathrm{z}$ of 637 .

\section{DISCUSSION}

Rapid identification of solanesol even in the presence of a complex matrix can be accomplished with $\mathrm{FAB}$ mass

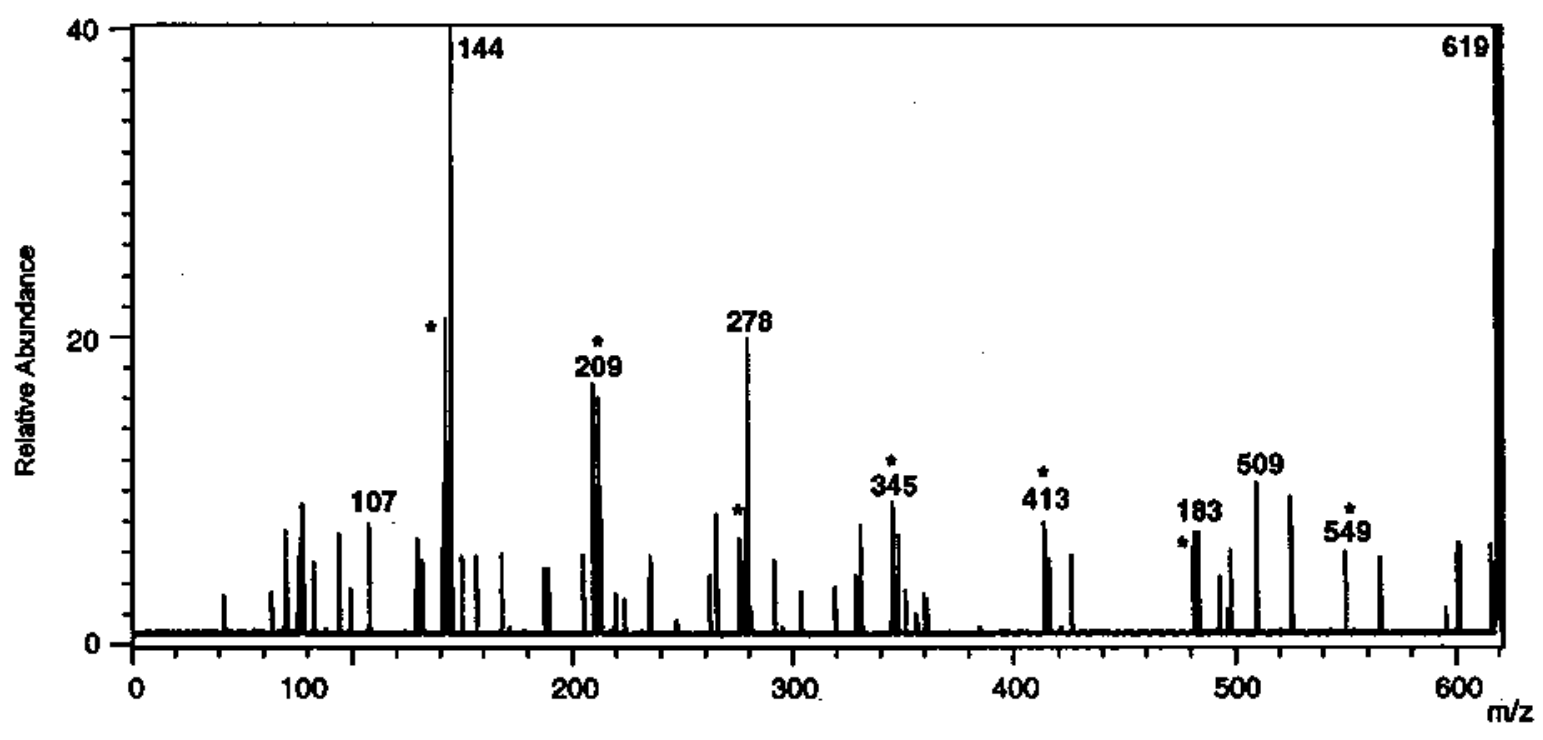

Figure 4.

FAB MSMS/MS spectrum of the ions derived from the first generation product ion of $\mathrm{m} / \mathrm{z}$ of 619 whlch was formed from the parent lon of $\mathrm{m} / \mathrm{z}$ of 637 . 
spectral analysis using a lithium dopant: This can be applied to investigations which require expedient screening for solanesol. The $(M+L i)^{+}$ion of $m / z$ of 637 which appears in the presence of lithium dopant but not in the undoped sample is a very characteristic marker for the presence of solanesol which is quite sufficient for most screening analyses. Confirmation of the identity of the ion as lithiated solanesol can be accomplished an MS/MS experiment which, as Figure 2 shows, yields a characteristic fingerprint spectrum for the entire terpene chain of solanesol. This qualitative detection of solanesol can be accomplished from a complex matrix such as a crude extract or from paper; however, matrix effects limit the analysis to qualitative information.

Although the initial goal of the research was to develop a facile method for detection of solanesol, the ambient temperature, soft ionization, direct probe analysis revealed that what was assumed to be "purified" solanesol was really a mixture. Exact mass assignment and comparison of the information gained from the MS/MS analysis of the unknown components, which yielded spectra containing information on the entire alkyl chain, with the MS/MS spectrum of solanesol permitted structural characterization of these components without further separation or purification.

$F A B$ in combination with tandem methods not only permitted structural characterization of solanesol and related compounds, but application of the MS/MS/MS experiment provided insight into the mechanism of fragmentation. Although the MS/MS/MS analysis is somewhat limited in sensitivity, it does provide a very viable alternative in many cases to the use of labeled materials for confirmation of structural assignment from spectral data.

\section{REFERENCES}

1. Tomer, K.B.: FAB/MS/MS for the determination of biomolecules: a compendium; Mass Spectrom. Rev. 8 (1989) 483-511.

2. Beimann, K.: Mass spectrometric determination of the amino acid sequence of peptides and proteins; Mass Spectrom. Rev. 6 (1987) 1-76.

3. Gross, M.L.: Charge-remote fragmentations: method, mechanism and applications; Int. J. Mass Spectrom. Ion Proc. 118/119 (1992) 137-165.

4. Matsuda, H.: High-resolution high transmission mass spectrometer; Int. J. Mass Spectrom. Ion Proc. 66 (1985) 209-215.

5. Adams, J.: Charge-remote fragmentation: analytical application and fundamental studies; Mass Spectrom. Rev. 9 (1990) 141-186.

6. Jensen, N. J., Tomer, K. B., and M.L. Gross: Gasphase ion decompositions occurring remote to the charge site; J. Am Chem. Soc. 107 (1985) 1863-1868.

Autbors' address

Philipp Morris Research Center,

P. O. Box 26583,

Richmond, Virginia,

23261-6583, USA. 